\title{
Barreras percibidas contra la implementación en los servicios de urgencias hospitalarios en España de un protocolo de cribado de consumo de alcohol e intervención breve en adolescentes
}

\author{
Perceived barriers to implementing screening and \\ brief intervention for alcohol consumption by adolescents \\ in hospital emergency department in Spain
}

\begin{abstract}
María Falcón*, Javier Navarro-Zaragoza*, Rosa María García-Rodríguez*,**, Daniel Nova-López**, María Dulce González-Navarro***, María Mercadal**********, Óscar García-Algar*********, Aurelio luna Ruiz-Cabello*.

*Departamento de Ciencias Sociosanitarias, Medicina Legal y Forense, Universidad de Murcia; **Hospital Virgen de la Arrixaca, Murcia; ***Hospital Virgen del Castillo, Yecla, Murcia; ****Servicio de Pediatría, Hospital del Mar, Barcelona; *****Departamento de Pediatría, Obstetricia y Ginecología, Universidad Autónoma, Barcelona.
\end{abstract}

\section{Resumen}

Introducción: El cribado de consumo de alcohol en menores está ampliamente justificado en el ámbito sanitario por la evidencia epidemiológica de consumo y por la especial vulnerabilidad de este colectivo, que además se inicia a edades muy tempranas e ingiere grandes cantidades. Los servicios de urgencias (ED) podrían ser un entorno donde realizar la detección precoz e implementar una intervención breve (IB) por parte de los profesionales.

Objetivo: El objetivo de este estudio es conocer las barreras percibidas por los profesionales sanitarios para implantar con éxito en los servicios de urgencias hospitalarios un protocolo de detección e IB en menores. Material y métodos: Análisis cualitativo exploratorio mediante grupos focales con preguntas semiestructuradas, flexibles y abiertas para conocer las creencias, actitudes y barreras percibidas por los profesionales de los centros donde se desarrollará un proyecto de cribado de consumo de alcohol e IB en adolescentes que acuden a Urgencias.

Resultados: Las principales barreras percibidas fueron falta de tiempo, sobrecarga de trabajo, desconfianza en la sinceridad de las respuestas, necesidad de protocolos estandarizados de trabajo, desconocimiento de herramientas de cribado validadas y sencillas, falta de entrenamiento/concienciación y dudas médico-legales sobre el consentimiento informado y la confidencialidad del menor.

Conclusiones: Las barreras percibidas para implementar la herramienta de cribado e IB son similares a las descritas por otros autores y sería necesario mejorar la organización de los circuitos asistenciales, no limitar los recursos dedicados a la atención en urgencias y favorecer la motivación y la formación de los profesionales.

Palabras clave: Adolescentes; Cribado; Urgencias; Alcohol; Intervención breve; Barreras percibidas.

\begin{abstract}
Background: Screening for alcohol consumption in adolescents is widely justified in the health care field because of the particular vulnerability of this population, which starts drinking alcohol at a very early age and frequently consumes high levels of the same. Hospital emergency departments (ED) could be a good venue to manage early detection and carry out brief intervention (BI) programmes.

Objectives: The aim of this study was to identify perceived barriers for medical staff of three hospitals in Spain to successfully implement a protocol for alcohol detection and BI for minors in the ED.

Methods: Exploratory qualitative analysis using focus groups with semistructured, flexible and open-ended questions to explore beliefs, attitudes, and barriers perceived by professionals to screening alcohol consumption and implementing BI in adolescents attended at the ED. Results: The main perceived barriers by health professionals were lack of time, work overload, mistrust, lack of validated and simple screening tools, lack of training/awareness and legal concerns about informed consent and confidentiality.

Conclusions: Barriers to screening and intervention in ED are similar to those described previously. It is necessary to improve organization of time allocated for medical consultations, avoid limiting ED resources, motivate staff and provide appropriate training.

Keywords: Adolescents; Screening; Emergency department; Alcohol; Brief intervention; Perceived barriers.
\end{abstract}


$\mathrm{E}$ 1 abuso de las sustancias es un problema social grave, con consecuencias importantes sociales, sanitarias y económicas. En particular, los adolescentes están entre los grupos de edad más vulnerables, caracterizados por cambios neuroconductuales con un rápido desarrollo de los sistemas límbicos y una inmadurez relativa de los sistemas cognitivos que puede fomentar el comportamiento de riesgo y el uso de sustancias. Ciertamente, la adolescencia representa un periodo específico de vulnerabilidad en cuanto al inicio del uso de sustancias y de transición al abuso y la dependencia de sustancias (Guerri y Pascual, 2010; Rutherford, Mayes y Potenza, 2010). Es sabido que el consumo de alcohol antes de la adultez puede afectar el desarrollo neurocognitivo y resultar en alteraciones físicas, psicológicas y sociales. Hay una asociación entre el consumo y accidentes de tráfico, homicidios, suicidios, contactos sexuales precoces, fracaso escolar, enfermedad mental y delincuencia, entre otros (Kaul y Coupey, 2002). En este sentido, algunos investigadores especulan que la edad del primer uso de sustancias puede suponer un factor de riesgo de la vulnerabilidad subyacente a la drogodependencia, y que con frecuencia resulta en una probabilidad mayor de aumentar la cantidad y la variedad de las sustancias consumidas (Chen, Storr y Anthony, 2009).

Por esta razón, la Estrategia Nacional sobre Drogas (2009-2016) (Plan Nacional sobre Drogas, 2009) anima a los médicos de atención primaria, los ED y los especialistas médicos a participar en la detección temprana de fumadores, abusadores de alcohol, usuarios de drogas psicoactivas y consumidores de drogas ilícitas, y en la implementación de terapia e IB. También contempla planes para desarrollar proyectos de investigación epidemiológicos, clínicos, básicos y sociales con menores.

El cribado del consumo de alcohol, tabaco y el abuso de drogas es ampliamente justificado en grupos de riesgo, tales como menores, por razones que incluyen su vulnerabilidad (Guerri y Pascual, 2010; Rutherford, Mayes y Potenza, 2010) y evidencia de consumo (ESTUDES 2010). Los adolescentes tienen sus propias expectativas y motivaciones respecto del consumo de alcohol (Monk y Heim, 2016; Mezquita, Stewart, Kuntsche y Grant, 2016) y la mayoría de las conductas de riesgo permanecen desconocidas tras una consulta médica. Es por esta razón que muchos investigadores han argumentado a favor de aplicar herramientas estructuradas de cribado adecuadas para la prevención de conductas problemáticas (Tiburcio Sáinz et al., 2016; García Carretero, Novalbos Ruiz, Martínez Delgado, y O'Ferrall González, 2016; Hidalgo Vicario y Redondo Romero, 2007; Committee on Substance Abuse, 2011). El modelo SBIRT de cribado, IB y derivación a tratamiento puede ser adecuado para la identificación e intervención en el caso de adolescentes en riesgo de desarrollar trastornos de uso de sustancias y aquellos cuyo uso de sustancias les pone en riesgo. El esfuerzo considerable de facilitar información y for- mación sobre este tema en las escuelas requiere de refuerzo en los servicios sanitarios, donde el mensaje ha de tener un apoyo en información clara y terapia para los pacientes. Los adolescentes no acuden a los servicios de atención primaria con la misma frecuencia que acudían a los servicios pediátricos antes de cumplir los catorce años de edad. Es más, el uso de alcohol y drogas está relacionado con muchos factores, incluso rasgos de personalidad (Gonzálvez, Espada, Guillén-Riquelme, Secades y Orgilés, 2016) y cuando los adolescentes empiezan a usar drogas recreativas carecen de señales o síntomas que les lleve a la consulta médica, y sólo unos pocos acuden como resultado de su abuso o dependencia. Por lo tanto, el único contacto entre los adolescentes y los servicios sanitarias es en los ED. Por este motivo, representan una oportunidad crítica y única para iniciar cuidados enfocados en el abuso de alcohol y sustancias.

Ya se ha mostrado que el cribado y la IB están entre las diez mejores estrategias de salud pública para la prevención de problemas relacionados con el abuso de alcohol (Babor, 2003). Algunos estudios (Armitage, Rowe, Arden y Harris, 2014; Kelly, Donovan, Chung, Bukstein y Cornelius, 2009; Newton et al., 2011; Cunningham et al., 2012) han obtenido resultados buenos en la IB con grupos de pacientes diferentes, lo que ha resultado en un descenso significativo de problemas relacionados con el consumo y abuso de alcohol, especialmente a corto plazo.

Por todas las razones mencionadas, este proyecto se diseñó para estructurar, validar e implementar un modelo de cribado y de IB en los ED que pueda ser útil a los profesionales sanitarios para detectar el uso/abuso temprano de alcohol en menores. En el contexto de un proyecto más amplio, primero diseñamos un estudio cualitativo para analizar las creencias y las actitudes de los médicos en los ED hacia la prevención del consumo de alcohol en adolescentes, y para abordar su posible percepción de las barreras y los facilitadores existentes en cuanto a la introducción del cribado y la IB en su rutina diaria.

\section{Métodos}

Se realizó un análisis cualitativo y exploratorio mediante entrevistas y grupos focales entre enero y marzo del 2012. Los participantes fueron reclutados de entre el personal de los ED de tres hospitales ya involucrados en el proyecto de investigación: (H1) Hospital Virgen del Castillo en Yecla, Murcia (sureste de España), que atiende una población principalmente de entornos semirural y rural, equipado con 100 camas; Hospital Virgen de la Arrixaca, Murcia (H2) y Hospital del Mar, Barcelona (H3), que atienden zonas principalmente urbanas y diversas en términos económicos y étnicos, y que están considerados entre los mejores veinte hospitales en España con 1000 y 500 camas, respectivamente. Primero, los supervisores de los tres ED fueron invitados a participar en entrevistas de treinta minutos para desarro- 
llar una guía para implementar los grupos focales, basado en la temática descrita por Millstein y Marcell (2003), que incluyó preguntas abiertas semi-estructuradas sobre la percepción de los médicos del uso/abuso de alcohol, las características de su práctica, la provisión de servicios primarios para el abuso-prevención de uso de alcohol, tales como el cribado o la IB, habilidades, actitudes y creencias, y, finalmente, las barreras y los facilitadores (Tabla 1).

Después, los médicos ya involucrados en el proyecto, que representaban el 100\%, $25 \%$ y $30 \%$ del personal total de ED de H1, H2 y H3, fueron invitados y dieron su consentimiento para participar en grupos focales $(n=24)$. Estos grupos estaban compuestos de 11 Asistentes Médicos (H1) con entre 7-28 años de experiencia, cuatro de ellos mujeres, 7 Asistentes Médicos (H1) con entre 6-23 años de experiencia, con sólo un hombre, y 3 Asistentes Médicos (H3) y 3 residentes de medicina en su último año de carrera con entre 4-25 años de experiencia, cuatro de ellos mujeres. Los médicos que declinaron participar en el estudio citaron la falta de tiempo, u otro motivo similar, como justificación. Tres sesiones de 45 minutos se realizaron con los grupos focales, empleando un formato parecido al anteriormente mencionado. Dichas entrevistas y sesiones fueron grabadas íntegramente y transcritas textualmente después de obtener el consentimiento, y finalmente analizadas según la metodología de la teoría fundamentada (Glaser y Straus, 1967) por dos observadores independientes que tomaron nota de los temas centrales que surgieron. Sus notas fueron comparadas y las discrepancias resueltas mediante consenso. Cada investigador tomó la iniciativa para identificar subtemas. Una vez codificado, empleamos un método analítico sistemático e iterativo, basado en el método comparativo constante. Las citas directas seleccionadas para ilustrar los temas mencionados por los participantes son indicativas tanto de las respuestas típicas como de la diversidad en los puntos de vista obtenidas.

Tabla 1. Cuestionario y grupos focales.

\begin{tabular}{|c|c|}
\hline Preguntas directas & $\begin{array}{l}\text { Esquema a seguir, } \\
\text { según la respuesta }\end{array}$ \\
\hline $\begin{array}{l}\text { ¿Cómo percibe el consumo de alcohol y otras sustancias por parte } \\
\text { de adolescentes en tu área? }\end{array}$ & $\begin{array}{l}\text { - Cuantitativo } \\
\text { - Cualitativo }\end{array}$ \\
\hline $\begin{array}{l}\text { ¿Hace cribado (detección temprana) del consumo de alcohol en } \\
\text { adolescentes como parte de su rutina diaria? }\end{array}$ & $\begin{array}{l}\text { - Nunca } \\
\text { - Solo en casos con síntomas de intoxicación aguda } \\
\text { - Como parte de mi rutina diaria }\end{array}$ \\
\hline $\begin{array}{l}\text { Una vez identificado el consumo, ¿desarrolla algún tipo de } \\
\text { intervención? }\end{array}$ & $\begin{array}{l}\cdot \text { Sí } \\
\cdot \text { No }\end{array}$ \\
\hline \multirow[t]{2}{*}{$\begin{array}{l}\text { IB - ¿Cómo lo implementa en la práctica? } \\
\cdot \text { ¿Deriva al paciente a otro centro de salud diferente? } \\
\cdot \text { ¿Cuál? } \\
\cdot \text { ¿Avisa a los padres? }\end{array}$} & $\begin{array}{l}\text { - Entrevista motivacional } \\
\text { - Terapia Breve }\end{array}$ \\
\hline & $\begin{array}{l}\text { - Pediatra } \\
\text { - Centro de rehabilitación } \\
\text { - Otros }\end{array}$ \\
\hline \multicolumn{2}{|l|}{$\begin{array}{l}\text { ¿Se considera con la formación para detectar si los adolescentes } \\
\text { tienen problemas con el uso/abuso de alcohol? }\end{array}$} \\
\hline \multicolumn{2}{|l|}{$\begin{array}{l}\text { ¿Se considera con la formación para detectarlo usando una } \\
\text { herramienta estandarizada? }\end{array}$} \\
\hline \multicolumn{2}{|l|}{$\begin{array}{l}\text { ¿Se considera con la formación para implementar una intervención } \\
\text { breve? }\end{array}$} \\
\hline \multicolumn{2}{|l|}{$\begin{array}{l}\text { ¿Piensa que sería de utilidad realizar cribados como parte de la } \\
\text { rutina diaria de los servicios de ED? }\end{array}$} \\
\hline ¿Cuáles barreras tendría que afrontar? & $\begin{array}{l}\text { - Falta de tiempo/recursos humanos } \\
\text { - Dudas respecto de mi responsabilidad en el asunto } \\
\text { - Dudas respecto de la utilidad del cribado y de la IB } \\
\text { - Preocupación sobre las implicaciones legales y del consentimiento informado } \\
\text { o la confidencialidad en las intervenciones con menores y sus tutores } \\
\text { - Falta de conocimiento/habilidad con herramientas de cribado } \\
\text { - Falta de cooperación/sinceridad de las respuestas de los menores } \\
\text { - Falta de formación/habilidades específicas en el trato con adolescentes } \\
\text { - Falta de procedimientos estandarizados }\end{array}$ \\
\hline Facilitadores & \\
\hline
\end{tabular}




\section{Resultados}

\section{Percepción del consumo de alcohol}

Los médicos consultados desconocían las estadísticas sobre el consumo de alcohol en España, pero, en general, tenían la percepción de que la prevalencia del consumo de alcohol era alta. Los miembros de GF2 estaban especialmente preocupados por los adolescentes que bebían grandes cantidades de alcohol de manera compulsiva y exagerada ('binge drinking'): «un problema generalizado, en particular entre los adolescentes de mayor edad, relacionado con su tiempo libre durante los fines de semana, festivos o fiestas de cumpleaños, cuando incluso los consumidores no-habituales celebran mediante la ingesta de alcohol», con «la gran cantidad que beben» como tema central.

\section{Experiencia previa con cribado}

Los participantes únicamente preguntaban a los pacientes y a sus acompañantes sobre su consumo de alcohol cuando parecían estar intoxicados, habían sufrido un accidente de tráfico, mostraban cambios en su comportamiento, o estaban inconscientes: «Preguntamos a los pacientes y a sus acompañantes cuando parece que están ebrios (...), y habitualmente de manera separada, como en casos de intoxicación etílica aguda, cuando normalmente vienen con amigos que nos dicen lo que han bebido».

Es importante subrayar que, exceptuando a los dos médicos de FG3 (Barcelona), los participantes desconocían o no usaban herramientas de cribado estructuradas autoinformadas, como CRAFFT, CAGE o AUDIT.

\section{Experiencia previa con IB}

Los médicos consultados no realizaban entrevistas motivacionales, sino que únicamente ofrecían terapia breve en casos de intoxicación etílica aguda: «Normalmente les explico los riesgos del consumo a medio y largo plazo, especialmente en los casos de adolescentes que beben grandes cantidades», "No en casos de adolescentes que vienen ligeramente ebrios que han tenido una pelea para poder denunciar su lesión, son casos en los que son dados de alta rápidamente y no suelen cooperar».

Cuando se les preguntaba acerca de sus habilidades en la gestión de uso/abuso de sustancias/alcohol entre adolescentes, los médicos mencionaron que derivaban los pacientes a Atención Primaria: «Suelo recomendarle al paciente que pida una cita con su pediatra», «Les derivamos a Atención Primaria porque es el procedimiento correcto para el tratamiento de enfermedad mental. No se nos permite derivar a los pacientes a los departamentos de salud mental desde los ED, únicamente en casos realmente urgentes».

Respecto de la información que ofrecen a los padres, encontramos actitudes diferentes: los participantes muchas veces no pueden informar a los padres o tutores por- que «acuden solos o con amigos», «Sólo podemos alertar a los padres si son menores de edad para dar su consentimiento», «los padres del adolescente ya saben si bebe».

\section{Conocimiento y habilidades en el uso de cribado}

Primero, se les preguntó a los médicos sobre su habilidad para detectar problemas relacionados con el uso/ abuso de alcohol entre adolescentes, y el análisis de los resultados apunta hacia dos ideas opuestas: a) se consideran capacitados para identificar dichos problemas entre adolescentes: «Sí, nos damos cuenta que tienen problemas con el alcohol o con otras drogas adictivas», o b) no se consideran capaces de identificar problemas con el alcohol y opinan que tampoco es su responsabilidad hacerlo: «No, nuestra obligación como médico es diagnosticar, y descartar, estabilizar o tratar las patologías cuanto antes para poder tratar a otros pacientes que llegan». Cuando modificamos y reformulamos la pregunta y preguntamos a los participantes si se consideraban capaces de detectar el uso/abuso de alcohol entre adolescentes usando una herramienta objetiva y estructurada, todos los participantes, menos dos de Barcelona, contestaron que no conocían ninguna herramienta validada de cribado como AUDIT, CAGE o CRAFFT. También consideraron que serían capaces de aplicarlas si recibiesen la formación adecuada: «Sí, pero mientras no afecte seriamente a nuestro trabajo».

\section{Conocimiento y habilidades en el uso de IB}

Todos los participantes se consideran formados y cómodos con la terapia breve, pero en ocasiones reconocían la necesidad de tener más formación en la entrevista motivacional. «Sí, la mayoría de nosotros somos médicos generales, lo que supone una ventaja en la prevención y la promoción de la salud», «Ofrecemos intervención y terapia breves exclusivamente».

Cuando se les preguntó si sería útil aplicar un cribado autoinformado o IB como parte de la rutina diaria en los ED, la mayoría de los participantes opinó que sería posible únicamente cuando los adolescentes llegaban con una intoxicación etílica aguda, aunque estaban de acuerdo sobre las ventajas del cribado: «¿Útil? Sí, claro, pero en caso de diagnosticar comportamiento de riesgo, la intervención se podría realizar en cualquier lugar», «Sí, pero sólo si no fuese perjudicial para pacientes en situaciones críticas». Varios de los médicos consideraban que el cribado no era parte de su trabajo, y manifestaron muchas dudas respecto de su responsabilidad en relación a este tema: «Cuando la visita médica no está relacionada con el consumo de sustancias, sería muy extraño centrar la atención en este tema», «Bueno, en realidad los pediatras deberían hacerse cargo de esto».

\section{Barreras}

Dos barreras importantes a la efectividad de este programa parecían ser falta de tiempo y de recursos humanos: 
«Por ejemplo, ahora estamos desbordados con un brote de influenza», «Hay momentos en los cuales sería literalmente imposible». Es más, esta sensación es recurrente en todas las respuestas sobre la viabilidad de implementar cribado e IB en los ED: «Si no acorta la atención a los pacientes en situaciones de gravedad», «Si no requiere mucho tiempo», «Si tenemos bastante tiempo», «Si es una noche poco ajetreada», «Si no estamos con sobrecarga de trabajo», etc. También, expresaron el temor de cómo reaccionarían los padres al saber del cribado de consumo de alcohol en menores, y surgían dudas acerca de la necesidad de obtener el consentimiento de los padres. "Puede que los padres se molesten si preguntamos a sus hijos sobre el uso de sustancias». No fueron motivo de preocupación la escasez de instrumentos de cribado o el conocimiento y las habilidades de los médicos en el uso de cribado e IB, ni tampoco los participantes de este estudio los percibieron como barreras. Es más, pensaron que su implementación sería sencilla, una vez recibiesen formación. Por último, otras de las barreras mencionadas reflejaron los sentimientos de los médicos, su falta de implicación o sensibilidad respecto de este tema, que habitualmente justificaban por la falta de tiempo o veracidad de las respuestas de los adolescentes: «Algunos de los pacientes dan respuestas falsas, así que no queremos perder el tiempo».

\section{Facilitadores}

La mayoría de los facilitadores percibidos fueron expresados como necesidades y reportados al finalizar las entrevistas y los grupos focales. Se consideró básico asegurar la confidencialidad adecuada para los pacientes, y establecer confianza entre pacientes y médicos. Se mencionó un entorno adecuado, instrumentos estructurados fiables, sencillos y cortos, y formación en el tratamiento de adolescentes con problemas relacionados con el uso/abuso de alcohol y sustancias.

Uno de los médicos mencionó la autoridad de dicho puesto en los ED, que probablemente difería de los puestos de los padres y los profesores, percibiéndolo como una ventaja que podría resultar en mayor probabilidad de obtener respuestas honestas, dada la relación personal de mayor cercanía existente entre los médicos de atención primaria/padres/profesores y los adolescentes.

De la misma manera, los hospitales se definieron como ideal: «Cuando acuden debido a embriaguez, se sienten avergonzados y asustados cuando se les da el alta, y quizás eso puede ser útil para cualquier intervención».

\section{Discusión}

Los participantes percibieron el consumo de alcohol entre adolescentes como un problema muy serio, debido principalmente a las grandes cantidades bebidas. No obstante, desconocían la prevalencia del consumo en España que, quizás, requiere mayor difusión. Únicamente se indagaba acerca del consumo de alcohol de los adolescentes en situaciones de intoxicación etílica aguda, lo que representa una oportunidad perdida. Dicho cribado conlleva preguntas directas a los pacientes y sus acompañantes sobre su consumo. Esto es una equivocación, dado que se ha mostrado que el consumo autoinformado por parte de los adolescentes puede estar escasamente reportado, particularmente si los adolescentes acuden acompañados de sus padres y están preocupados por motivos de confidencialidad, un probable ingreso por riesgo de uso de sustancias, o por ansiedad como consecuencia del descubrimiento de sus padres de su uso de sustancias, lo que podría explicar por qué los adolescentes prefieren simplemente decir «No uso» (Delaney-Black et al., 2010; Elliot y Larson, 2004). Existen instrumentos de cribado estructurados como AUDIT, CAGE o CRAFFT que han mostrado propiedades discriminatorias buenas para determinar la presencia de trastornos de uso de alcohol en adolescentes entre las edades de 14-18 años en atención primaria o ED (Kelly, Donovan, Chung, Bukstein y Cornelius, 2009; Newton et al., 2011). Esto es coherente con un estudio que mostró que ningún médico usaba CRAFFT, un instrumento de cribado adecuadamente validado para el uso de alcohol entre adolescentes y que una quinta parte de los médicos identificaban el consumo de alcohol entre adolescentes usando el cuestionario CAGE, una herramienta de cribado usada con frecuencia en la población adulta (Gordon, Ettaro, Rodríguez, Mocik y Clark, 2011). Por esto, quizás debería hacerse mayor hincapié en la relación entre alcohol y adolescentes durante la formación médica, como han sugerido estudios previos (Danielson, Rivara, Gentilello y Maier 1999; Miller, Naimi, Brewer y Jones, 2007). Se ha reportado con anterioridad que la falta de recursos y de sensibilización afectan negativamente el cribado rutinario (Matali et al., 2009).

Por otro lado, hay otras maneras de estimar el consumo de alcohol, como los biomarcadores de etanol (etil-glucurónido) en pelo, pero estos análisis son costosos y requieren de un periodo largo de pruebas con muestras antes del tratamiento, algo no siempre disponible en los ED. Es más, el análisis de las uñas también se considera comparable y, a veces, superior para dichas mediciones. No obstante, los biomarcadores de etanol solo son fiables en casos de consumo de alcohol en grandes cantidades, lo que no refleja el perfil del comportamiento adolescente (Morini, Politi y Polettini, 2009).

En lo que se refiere a la metodología de intervención, la terapia breve fue la única implementada, aunque un estudio anterior propuso otras acciones más complejas, como la entrevista motivacional, que resultaban en un uso de alcohol significativamente menor en el seguimiento realizado a un año (Clark y Moss, 2010). La mayoría de los médicos contestó que consideraba que tenía la habilidad para implementar la entrevista motivacional, en co- 
herencia con estudios previos sobre la necesidad de los recursos humanos en la atención sanitaria de más tiempo, seguimiento y formación. También, se necesita de más estudios, como el de Monti et al. (1999), que comprueben la eficacia de las intervenciones realizadas por parte de médicos diferentes.

Nuestro estudio comprobó que todos los participantes derivaron a sus pacientes a Atención Primaria e informaron a sus padres o tutores, si estaban en el momento del alta, según la edad del menor. Hallazgos recientes indicaron, en un seguimiento de seis meses, que el grupo que recibió IB con sus padres obtuvo resultados significativamente mejores en cuanto al uso de alcohol, ingesta compulsiva y exagerada de alcohol y días de uso de sustancias, comparado con el grupo que solo recibió una valoración, y menos días de ingesta de alcohol comparado con el grupo que solo recibió IB (Mitchell, Gryczynski, O’Grady y Schwartz, 2013).

Varios estudios proponen que la entrega de servicios de prevención relacionados con el alcohol para adolescentes varía en función de las actitudes y las creencias de los médicos respecto de la prevención y la práctica. Además, aquellos con creencias más positivas sobre la importancia de la prevención están de acuerdo con el cribado a edades tempranas y se sienten más seguros en sus habilidades de tratar el uso de alcohol con adolescentes durante el cribado (Marcell, Halpern-Felsher, Coriell y Millstein, 2002). Según nuestros resultados, la viabilidad de implementar cribado e IB dependía de las barreras y facilitadores diferentes percibidos por nuestros participantes, que detallaremos a continuación.

En coherencia con estudios anteriores (Millstein y Marcell, 2003; Aristeiguieta, 2000), se mencionaron varias «barreras»: falta de tiempo, sobrecarga de trabajo por motivos estacionales, desconfianza en la sinceridad de las respuestas, falta de formación/sensibilización y preocupaciones legales en relación al consentimiento informado y la confidencialidad. Por lo contrario, la escasez de protocolos estandarizados de trabajo, y las habilidades y el conocimiento de los médicos con el cribado y la IB diferían de estudios previos (Beich, Gannik y Malterud, 2002); estos aspectos no se percibieron como barreras al considerar que era sencilla su puesta en práctica tras una formación adecuada. Estas barreras son iguales a las mencionadas con anterioridad por otros autores en distintos entornos del sector sanitario para la implementación correcta del SBIRT (Gordon, Ettaro, Rodríguez, Mocik y Clark, 2011). La formación es un tema crítico, dado que hallazgos anteriores destacan que los médicos de Atención Primaria con formación sobre el alcohol y el cribado aplican el SBIRT en su rutina diaria con una mayor frecuencia considerable (Ozer et al., 2011). Quizás una de las barreras que menos atención recibe es el desconocimiento de temas legales relacionados con el consentimiento informado y la confi- dencialidad, como han señalado estudios previos que postulan que algunos médicos piensan que preguntar a los pacientes sobre su consumo de alcohol es una invasión de su privacidad (Bradley, Boekeloo y Novik, 2005; Schermer et al., 2003). Esto podría estar relacionado con la naturaleza de este entorno sanitario, que trata más las intoxicaciones agudas que la promoción de salud.

Por último, debemos señalar que los costes económicos y su reembolso no se consideraron barreras en nuestro estudio, lo contrario a la literatura consultada sobre la implementación de SBIRT en distintos países. Opinamos que esto podría resultar del sistema sanitario libre y universal disponible en la actualidad en España (Danielson, Rivara, Gentilello y Maier 1999; Aristeiguieta, 2000; Schermer et al., 2003).

Es de gran importancia que los médicos sean conocedores de los patrones de uso-abuso de alcohol y otras sustancias por adolescentes con el fin de alertar a sus pacientes sobre las consecuencias en todos los entornos sanitarios. Las barreras percibidas contra la implementación en los ED en España de un protocolo de cribado y de IB detectadas por este estudio son similares a las mencionadas por otros autores (Platt et al., 2016) en los servicios de Atención Primaria, principalmente relacionados con la falta de tiempo y de recursos humanos y preocupación sobre las implicaciones legales en relación al consentimiento informado y la confidencialidad. No debe haber duda alguna que la prevención es responsabilidad de todos, y que es necesario luchar contra el escepticismo de algunos médicos sobre la capacidad de nuestro sistema para abordar las consecuencias del abuso de sustancias en la adolescencia.

Los adolescentes suelen frecuentar los ED en casos de intoxicación aguda, que debería ofrecer intervenciones para promover la abstinencia y prevenir 'binge drinking' (Kelleher, Renaud, Ehrlich y Burd, 2013). No obstante, acciones preventivas (e investigación) en los ED son difíciles porque los médicos se sienten presionados para ofrecer cuidados sanitarios, lo que puede limitar la recopilación de datos y también la ineficacia de las implementaciones de SBIRT llevadas a cabo (Van Hook et al., 2007).

Por ello, es necesario mejorar la distribución/organización de la atención sanitaria sin limitar los recursos alocados a los ED, para garantizar la motivación de los médicos y para involucrar a todos los recursos humanos de este sector. La derivación a tratamiento en los casos de sospecha de abuso de alcohol es otro punto clave que podemos destacar, y los historiales médicos electrónicos debería incluir datos sobre cribados y servir de apoyo en la continuidad del tratamiento, cuando necesario.

\section{Reconocimientos}

Este estudio ha sido parte de un proyecto financiado por el Plan Nacional sobre Drogas (2011-2013). 


\section{Conflicto de intereses}

Los autores declaran la inexistencia de conflicto de intereses. Todos los autores han contribuido hacia la versión definitiva de este artículo y aprobado la misma.

\section{Referencias}

Aristeiguieta, C. A. (2000). Screening patients for alcohol, tobacco, and other drug misuse: the role of brief interventions. Western Journal of Medicine, 172, 53-57.

Armitage, C. J., Rowe R., Arden M. A. y Harris P. R. (2014). A Brief Psychological Intervention That Reduces Adolescent Alcohol Consumption. Journal of Consulting and Clinic Psychology, 82, 546-550. doi:10.1037/a0035802.

Babor, T. (2003). Alcohol: no ordinary commodity. Oxford: Oxford University Press.

Beich, A., Gannik, D. y Malterud, K. (2002). Screening and brief intervention for excessive alcohol use: qualitative interview study of the experiences of general practitioners. British Medical Journal, 325, 870-874.

Bradley, O., Boekeloo, M. S. y Novik, G. (2005). Clinical Approaches to Improving Alcohol Education and Counselling in Adolescents and Young Adults. Journal of Trauma, 59, S137-143.

Chen, C. Y., Storr, C. L. y Anthony, J. C. (2009). Early-onset drug use and risk for drug dependence problems. Addiction Behaviour, 34, 319-322. doi:10.1016/j.addbeh.2008.10.021.

Clark, D. B. y Moss, H. B. (2010). Providing alcohol-related screening and brief interventions to adolescents through health care systems: obstacles and solutions. Plos Medicine, 7, e1000214. doi:10.1371/journal.pmed.1000214.

Committee on Substance Abuse, (2011). Substance Use Screening, Brief Intervention, and Referral to Treatment for Pediatricians. Pediatrics, 128, e1330-1340. doi:10.1542/peds.2011-1754.

Cunningham, R. M., Bernstein, S. L., Walton, M., Broderick, K., Vaca, F. E., Woolard, R.,... D'Onofrio, G. (2009). Alcohol, tobacco, and other drugs: future directions for screening and intervention in the emergency department. Academic Emergency Medicine, 16, 1078-1088. doi:10.1111/j.1553-2712.2009.00552.x.

Danielson, P. E., Rivara, F. P., Gentilello, L. M. y Maier, R. V. (1999). Reasons why trauma surgeons fail to screen for alcohol problems. Archives of Surgery, 134, 564-568.

Delaney-Black, V., Chiodo, L. M., Hannigan, J. H., Greenwald, M. K., Janisse, J. Patterson, G.,... Sokol, R. J. (2010). Just say "I don't": lack of concordance between teen report and biological measures of drug use. Pediatrics, 126, 887-893. doi:10.1542/peds.2009-3059.

Elliot, B. A. y Larson, J. T. (2004). Adolescents in midsized and rural communities: foregone care, perceived barriers, and risk factors. Journal of Adolescent Health, 35, 303-309. doi:10.1016/j.jadohealth.2003.09.015.
García Carretero, M. A., Novalbos Ruiz, J. P., Martínez Delgado, J. M. y O'Ferrall González, C. (2016). Validation of the Alcohol Use Disorders Identification Test in university students: AUDIT and AUDIT-c. Adicciones, 28, 194-204. doi:10.20882/adicciones.775.

Glaser B. G. y Strauss A. L. (1967). The discovery of grounded theory: strategies for qualitative research. Chicago: Aldine.

Gonzálvez, M. T., Espada, J. P., Guillén-Riquelme, A., Secades, R. y Orgilés, M. (2016). Association between personality traits and substance use in Spanish adolescents. Adicciones, 28, 108-115. doi:10.20882/adicciones.777.

Gordon, A. J., Ettaro, L., Rodriguez, K. L., Mocik, J. y Clark, D. B. (2011). Provider, patient, and family perspectives of adolescent alcohol use and treatment in rural settings. The Journal of Rural Health, 27, 81-90. doi:10.1111/ j.1748-0361.2010.00321.x.

Guerri, C. y Pascual, M. (2010). Mechanisms involved in the neurotoxic, cognitive, and neurobehavioral effects of alcohol consumption during adolescence. Alcohol, 44, 15-26. doi:10.1016/j.alcohol.2009.10.003.

Hidalgo Vicario, M. I. y Redondo Romero, A. (2007). Adolescentes y drogas. Un reto para los profesionales sanitarios. Evidencias en Pediatría, 3, 60.

Kaul, P. y Coupey, S. M. (2002). Clinical evaluation of substance abuse. Pediatric Review, 23, 85-94.

Kelleher, D. C., Renaud, E. J., Ehrlich, P. F. y Burd, R. S. (2013). Pediatric Trauma Society Guidelines Committee: Guidelines for alcohol screening in adolescent trauma patients: a report from the Pediatric Trauma Society Guidelines Committee. Journal of Trauma and Acute Care Surgery, 74, 671-682. doi:10.1097/TA.0b013e$31827 d 5 f 80$.

Kelly, T. M., Donovan, J. E., Chung, T., Bukstein, O. G. y Cornelius, J. R. (2009). Brief screens for detecting alcohol use disorder among 18-20 year old young adults in emergency departments: Comparing AUDIT-C, CRAFFT, RAPS4-QF, FAST, RUFT-Cut, and DSM-IV 2-Item Scale. Addiction Behaviour, 34, 668-674. doi:10.1016/j. addbeh.2009.03.038.

Marcell, A. V., Halpern-Felsher, B., Coriell, M. y Millstein, S. G. (2002). Physicians' attitudes and beliefs concerning alcohol abuse prevention in adolescents. American Journal of Preventive Medicine, 22, 49-55.

Matali, J. L., Pardo, M., Trenchs, V., Serrano, E., Gabaldón, S. y Luaces, C. (2009). Consumo de drogas en adolescentes. Dilema ético en el abordaje diagnóstico-terapéutico. Anales de Pediatría, 70, 386-390. doi:10.1016/j. anpedi.2008.12.006.

Mezquita, L., Stewart S. H., Kuntsche, E. y Grant, V. V. (2016). Cross-cultural examination of the five-factor model of drinking motives in Spanish and Canadian undergraduates. Adicciones, 28, 215-220. doi:10.20882/ adicciones.822. 
Miller, J. W., Naimi, T. S., Brewer, R. D. y Jones, S. E. (2007). Binge drinking and associated health risk behaviors among high school students. Pediatrics, 119, 76-85.

Millstein, S. G. y Marcell, A. V. (2003). Screening and counseling for adolescent alcohol use among primary care physicians in the United States. Pediatrics, 111, 114-122.

Mitchell, S. G., Gryczynski, J., O’Grady, K. E. y Schwartz, R. P. (2013). SBIRT for adolescent drug and alcohol use: Current status and future directions. Journal of Substance Abuse Treatment, 44(5), 463-472. doi:10.1016/j. jsat.2012.11.005.

Monti, P. M., Colby, S. M., Barnett, N. P., Spirito, A., Rohsenow, D. J., Myers, M., Woolard, R. y Lewander, W. (1999). Brief intervention for harm reduction with alcohol positive older adolescents in a hospital emergency department. Journal of Consulting and Clinical Psychology, 67, 989-994.

Monk, R. L. y Heim, D. (2016). Alcohol-related expectancies in adults and adolescents: similarities and disparities. Adicciones, 28, 35-40. doi:10.20882/adicciones.788.

Morini, L., Politi, L. y Polettini, A. (2009). Ethyl glucuronide in hair. A sensitive and specific marker of chronic heavy drinking. Addiction, 104, 915-920. doi:10.1111/ j.1360-0443.2009.02535.x.

Newton, A. S., Gokiert, R., Mabood, N., Ata, N., Dong, K., Ali, S.,... Wild, T. C. (2011). Instruments to detect alcohol and other drug misuse in the emergency department: a systematic review. Pediatrics, 128, e180-192. doi:10.1542/peds.2010-3727.

Ozer, E. M., Adams, S. H., Orrell-Valente, J. K., Wibbelsman, C. J., Lustig, J. L., Millstein, S. G.,... Irwin, C. E. (2011). Does delivering preventive services in Primary Care reduce adolescent risky behavior? Journal of Adolescent Health, 49, 476-482. doi:10.1016/j.jadohealth.2011.02.011.

Plan Nacional sobre Drogas. (2009). Estrategia Nacional sobre Drogas 2009-2016. Madrid, España: Delegación del Gobierno para el Plan Nacional sobre Drogas, Ministerio de Sanidad, Servicios Sociales e Igualdad.

Plan Nacional sobre Drogas. (2010). Encuesta estatal sobre uso de drogas en estudiantes de secundaria (ESTUDES). Madrid: Ministerio de Sanidad, Servicios Sociales e Igualdad. Recuperado de http://www.pnsd.msc.es/Categoria2/observa/pdf/ESTUDES_2010.pdf.

Platt, L., Meléndez-Torres, G. J., O’Donnell, A., Bradley, J., Newbury-Birch, D., Kaner, E. y Ashton, C. (2016). How effective are brief interventions in reducing alcohol consumption: do the setting practitioner group and content matter? Findings from a systematic review and metaregression analysis. BMJ Open, 6,: e011473. doi:10.1136/ bmjopen-2016-011473.

Rutherford, H. J., Mayes, L. C. y Potenza, M. N. (2010). Neurobiology of adolescent substance use disorders: implications for prevention and treatment. Child and
Adolescent Psychiatric Clinics of North America, 19, 479-492. doi:10.1016/j.chc.2010.03.003.

Schermer, C. R., Gentilello, L. M., Hoyt, D. B., Moore, E. B., Moore, J. B., Rozycki, G. S. y Feliciando, D. V. (2003). National survey of trauma surgeons' use of alcohol screening and brief intervention. Journal of Trauma, 55, 849-856.

Tiburcio Sainz, M., Rosete-Mohedano, G., Natera Rey, G., Martínez Vélez, N. A., Carreño García, S. y Pérez Cisneros, D. (2016). Validity and reliability of the Alcohol, Smoking, and Substance Involvement Screening Test (ASSIST) in university students. Adicciones, 28, 19-27. doi:10.20882/adicciones.786.

Van Hook, S., Harris, S. K., Brooks, T., Carey, P., Kossack, R., Kulig, J. y Knight, J. R. (2007). New England Partnership for Substance Abuse Research: The "Six T's": barriers to screening teens for substance abuse in primary care. Journal of Adolescent Health, 40, 456-461. 